\title{
ON THE LOCATION OF SINGULARITIES AT THE BOUNDARY OF THE DOMAIN OF OVERCONVERGENCE
}

\author{
G. A. READ
}

It is well known that the occurrence of gaps in a series and overconvergence are closely related phenomena; a classical theorem of Ostrowski states that a Taylor series which has gaps of relative leng th ${ }^{1}$ bounded below away from one has a sequence of partial sums overconvergent in the neighborhood of every regular point on the circle of overconvergence. If the relative length of the gaps tends to infinity, then the sequence of partial sums overconverges to the limit function in its region of regularity. We will improve on the latter result by showing that if the boundary of the region of overconvergence is sufficiently smooth, then the regions of overconvergence and regularity are identical, provided that the relative length of the gaps is greater than some finite number. We will also establish a criterion for the location of some of the singularities of the limit function when these two regions are not identical.

Consider a simply connected bounded schlicht domain $D$ containing $|z|<1$ but not the whole of $|z|=1$, and let $\mathcal{F}$ be the family ${ }^{2}$ of functions having a subsequence of partial sums of their Taylor expansion about the origin, overconvergent in $D$. Let $f(z)$ be an element of $\mathcal{F}$, and put $f(z)=\sum_{r=0}^{\infty} a_{r} z^{r}$ (necessarily of unit radius of convergence), $S_{n}(z)=\sum_{r=0}^{n} a_{r} z^{r}$ and $r_{n}(z)=f(z)-S_{n}(z)$. Suppose that the sequence $S_{n_{k}}$ overconverges in $\mathscr{D}$, and denote the complement of the closure of $D$ by $D^{*}$. Let $g(z)$ and $G(z)$ be the Green's functions of $D$ and $D^{*}$ with respect to the points 0 and $\infty$ respectively, then using the method of harmonic majorants we readily obtain the following estimates:

$$
\begin{aligned}
& \frac{1}{n_{k}} \log \left|r_{n_{k}}(z)\right|<-g(z)+\epsilon_{k}(z) \quad \text { if } z \in \mathfrak{D}, \\
& \frac{1}{n_{k}} \log \left|S_{n_{k}}(z)\right|<G(z)+\epsilon_{k}(z) \quad \text { if } z \in D^{*},
\end{aligned}
$$

Received by the editors January 29, 1965.

${ }^{1}$ If in the series $\sum a_{r} z^{r}, a_{r}=0$ for $n_{k} \leqq r \leqq \lambda n_{k}$, where $\lambda>1$ and $\left\{n_{k}\right\}$ is a monotonic increasing sequence of integers, then the series is said to have gaps of relative length $\lambda$.

${ }^{2} \mathcal{F}$ is not empty; see Bourion [1, Chapter 7]. 
where $\epsilon_{k}(z)$ denotes a sequence of functions of $z$ uniformly convergent to zero on compact sets of the given regions. ${ }^{3}$ If we let $z_{0}$ be a point of $\partial D$, the boundary of $D$, and $Q\left(z_{0}\right)$ be the set of all numbers $\delta$ for which the circle $\left|z-z_{0}\right|=\delta$ intersects $\partial \mathscr{D}$ in a set of zero measure, then if $\partial D$ is rectifiable we may find elements of $Q\left(z_{0}\right)$ which are as small as we please. If this were not so we could find a $\delta^{*}>0$ such that the intersection of $\partial \mathscr{D}$ with every circle $\left|z-z_{0}\right|=\delta, \delta<\delta^{*}$ is not of zero measure. Since $\partial D$ is rectifiable it follows that the circles $\left|z-z_{0}\right|=\delta, \delta<\delta^{*}$ are enumerable, which is a contradiction. If we let $\Gamma$ be the circle $\left|z-z_{0}\right|=\delta$ and put $\Gamma_{1}=\Gamma \cap D$ and $\Gamma_{2}=\Gamma \cap D^{*}$ we may then define a boundary characteristic by taking

$$
k\left(z_{0}, \delta\right)=\frac{\int_{\Gamma_{2}} G(z) d s}{\int_{\Gamma_{1}} g(z) d s}
$$

and then

$$
K\left(z_{0}, d\right)=\inf _{\delta<d}\left[k\left(z_{0}, \delta\right), \delta \in Q\left(z_{0}\right)\right] .
$$

We will show that if this characteristic $K\left(z_{0}, d\right)$ is less than one for all $d>0$ then the point $z_{0}$ must be a singularity of $f(z)$. This result is stated formally in the following theorem.

THEOREM 1. If $\partial \mathcal{D}$ is rectifiable and $K\left(z_{0}, d\right)<1$ for all $d>0$ then $z_{0}$ is a singularity of $f(z)$.

It is interesting to notice that $K\left(z_{0}, d\right)$ is independent of $f(z)$ and is dependent only on the geometry of $D$. In order to prove this result we require the following lemma which is a simple application of Poisson's formula.

Lemma. Let $\left\{u_{k}(z)\right\}(k=1,2 \cdots)$ be a sequence of functions subharmonic in the disc $\Omega:|z|<R$. Suppose that $u_{k}(z)<B$ in $\Omega(k=1,2 \cdots)$ for some constant $B$, and that

$$
U_{k}\left(\operatorname{Re}^{i \theta}\right)=\lim \sup u_{k}(z)\left(z \rightarrow \operatorname{Re}^{i \theta}, z \in \Omega\right)
$$

satisfies

$$
U_{k}\left(\operatorname{Re}^{i \theta}\right)<\epsilon_{k}
$$

for all $\theta(|\theta|<\pi)$ in a set $C_{k}$ of measure $\geqq 2 \pi-\delta_{k}$, where $\left\{\epsilon_{k}\right\}$ and

3 This notation will be used throughout, and $\left|\phi_{n}(z)\right|<\epsilon_{n}(z)$ on a set $\mathcal{E}$ will mean that $\phi_{n}(z) \rightarrow 0$ uniformly as $n \rightarrow \infty$ on compact subsets of $\mathcal{E}$. 
$\left\{\delta_{k}\right\}$ are sequences of positive numbers tending to zero. Then

$$
u_{k}(z)<\epsilon_{k}(z)
$$

in $\Omega$.

Proof. Let $|z|=r<R$. Then by the boundary harmonic majorant principle

$$
\begin{aligned}
u_{k}(z)< & \frac{1}{2 \pi} \int_{C_{k}} \frac{R^{2}-r^{2}}{R^{2}+r^{2}-2 R r \cos (\theta-\phi)} \epsilon_{k} d \phi \\
& +\frac{1}{2 \pi} \int_{\partial \Omega-C_{k}} \frac{R^{2}-r^{2}}{R^{2}+r^{2}-2 R r \cos (\theta-\phi)} B d \phi \\
& <\frac{\epsilon_{k}}{2 \pi} \int_{-\pi}^{\pi} \frac{R^{2}-r^{2}}{R^{2}+r^{2}-2 R r \cos (\theta-\phi)} d \phi+\frac{R+r}{R-r} \cdot \frac{B \delta_{k}}{2 \pi} \\
< & +\frac{2 R}{R-|z|} \frac{B \delta_{k}}{2 \pi}=\epsilon_{k}(z) .
\end{aligned}
$$

Proof of Theorem 1. Suppose that $f(z)$ is regular at $z=z_{0}$, then we may choose $\delta>0$ so that $f(z)$ is regular in $\left|z-z_{0}\right|<\delta$, and $k\left(z_{0}, \delta\right)<1$. From (1) and (2) we have

$$
\begin{array}{ll}
\left(1 / n_{k}\right) \log \left|r_{n_{k}}(z)\right|<-g(z)+\epsilon_{k}(z) & \text { if } z \in \Gamma_{1}, \\
\left(1 / n_{k}\right) \log \left|r_{n_{k}}(z)\right|<G(z)+\epsilon_{k}(z) & \text { if } z \in \Gamma_{2},
\end{array}
$$

but in addition ${ }^{4}$

$$
\left(1 / n_{k}\right) \log \left|r_{n_{k}}(z)\right|<B \quad \text { if } z \in \Gamma
$$

for some constant $B$. Let $V(z)$ be the function, harmonic in $\left|z-z_{0}\right|<\delta$ which takes the values $-g(z)$ on $\Gamma_{1}$ and $G(z)$ on $\Gamma_{2}$. Applying the lemma to the subharmonic functions $\left(1 / n_{k}\right) \log \left|r_{n_{k}}(z)\right|-V(z)$ and using (3), (4) and (5) we obtain

$$
\left(1 / n_{k}\right) \log \left|r_{n_{k}}(z)\right|<V(z)+\epsilon_{k}(z) \text { if }\left|z-z_{0}\right|<\delta .
$$

However,

$$
V\left(z_{0}\right)=\frac{1}{2 \pi \delta}\left\{\int_{\Gamma_{2}} G d s-\int_{\Gamma_{1}} g d s\right\}<0
$$

4 It is well known that $(1 / n) \log \left|r_{n}(z)\right|<\log |z|+\epsilon_{n}(z)$ on any set inside which $f(z)$ is regular (see for example Bourion $[1$, p. 8]). 
and $V(z)$ is continuous. It follows from (6) that $r_{n_{k}}(z) \rightarrow 0$ in some neighborhood of $z_{0}$, which implies that $z_{0}$ is not a boundary point of $D$, and this completes the proof of the theorem.

If in addition to knowing the region of overconvergence, we also know the relative length of the gaps in the series which appear after the partial sums $S_{n_{k}}(z)$, then we can improve on Theorem 1 in the following way. Suppose that $a_{r}=0$ for $n_{k}<r<N_{k}$ where $N_{k} / n_{k} \geqq \lambda>1$ for all $k$ sufficiently large, then we can replace (1) by

$$
\frac{1}{n_{k}} \log \left|r_{n_{k}}(z)\right|<-\lambda g(z)+\epsilon_{k}(z) \quad \text { if } z \in D \text { D. }
$$

THEOREM 2. If $\partial D$ is rectifiable and $K\left(z_{0}, d\right)<\lambda$ for all $d>0$ then $z_{0}$ is a singularity of $f(z)$.

The proof is identical to that of Theorem 1 with $g(z)$ replaced by $\lambda g(z)$ throughout.

ExAmple. Let $f(z)$ be any function which has a subsequence of

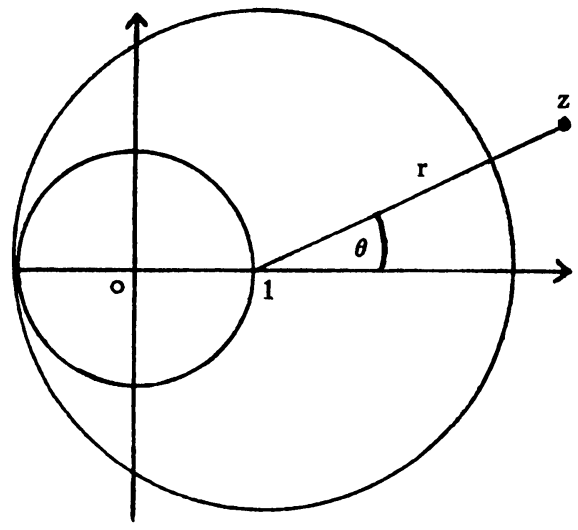

partial sums overconvergent in the disc $|z-1|<2$. Let the point $z=1$ be the origin for polar coordinates $r$ and $\theta$, then the Green's function of $D$ relative to $z=0$ is

$$
g(z)=\frac{1}{2} \log \frac{r^{2}+8 r \cos \theta+16}{r^{2}+2 r \cos \theta+1}-\log 2
$$

and

$$
\frac{\partial g}{\partial r}=\frac{r+4 \cos \theta}{r^{2}+8 r \cos \theta+16}-\frac{r+\cos \theta}{r^{2}+2 r \cos \theta+1} .
$$


The Green's function of $D^{*}$ relative to $z=\infty$ is $G(z)=\log (r / 2)$ and therefore

$$
\left(\frac{\partial g}{\partial r}\right)_{r=2}=\frac{-3}{8 \cos \theta+10} \quad \text { and } \quad\left(\frac{\partial G}{\partial r}\right)_{r=2}=\frac{1}{2} .
$$

Let $\gamma$ be the points of $|z-1|=2$ for which $\cos \theta<-\frac{1}{2}$, and let $z_{0}$ be a point of $\gamma$, then for all points of $D$ in some neighborhood of $z_{0}$, we have $-g(z)<G(z)$. Let $\Gamma$ be the circle $\left|z-z_{0}\right|=\delta$ then $\int_{\Gamma} G d s=0$ for all $\delta$, it follows that $\int_{\Gamma_{2}} G d s-\int_{\Gamma_{1}} g d s<0$ for $\delta$ sufficiently small, and therefore $K\left(z_{0}, d\right)<1$. We deduce from Theorem 1 that each of the points of the locus $\{|z-1|=2, x \leqq 0\}$ is a singularity of $f(z)$. If $\lambda>3$ then $-\lambda(\partial g / \partial r)_{r=2}>(\partial G / \partial r)_{r=2}$ and it follows from Theorem 2 that every point of $|z-1|=2$ is a singular point of $f(z)$.

Theorem 3. If $K\left(z_{0}, d\right)<\Lambda$ for all $z_{0} \in \partial D$ and all $d>0$ where $\Lambda$ is some positive constant, then every element $f(z)$ of $\mathcal{F}$ which has gaps of relative length $\Lambda$ after its sequence of partial sums which overconverge in $D$, has $\partial \mathcal{D}$ as a natural boundary.

The proof of this result is again similar to that of Theorem 1. The characteristic $K\left(z_{0}, d\right)$ will be uniformly bounded on $\partial D$ if this boundary is sufficiently smooth. For example, if we make the very restrictive assumptions that $D$ is bounded, $\partial D$ is rectifiable and has a tangent at every point, and that the interior and exterior mapping functions are regular on $\partial \mathcal{D}$, then it is not difficult to show that $K\left(z_{0}, d\right)$ is uniformly bounded. If $\partial / \partial n$ denotes differentiation along the outward normal on $\partial D$, then $\partial g / \partial n$ is uniformly bounded from zero and $\partial G / \partial n$ is uniformly bounded on $\partial D$, and we may estimate the integrals $\int_{\Gamma_{1}} g d s$ and $\int_{\Gamma_{2}} G d s$ in terms of these derivatives.

\section{BIBLIOGRAPHY}

1. G. Bourion, L'ultraconvergence dans les séries de Taylor, Actualités Sci. Indust., No. 472, Hermann, Paris, 1937.

2. A. J. Macintyre, An overconvergence theorem of G. Bourion, Ann. Acad. Sci. Fenn. Ser. A. I. no. 250/23 (1958), 11 pp.

3. - Size of gaps and regions of overconvergence, Collect. Math. 11 (1959), 165-174.

Woolwich Polytechnic, London 